\title{
Lung donor shortage - how to overcome it?
}

\author{
Bartłomiej Zych, Diana Garcia-Saez, Andre R. Simon
}

Department of Cardiothoracic Transplantation and Mechanical Circulatory Support, Harefield Hospital, Royal Brompton and Harefield NHS Foundation Trust, Harefield, United Kingdom

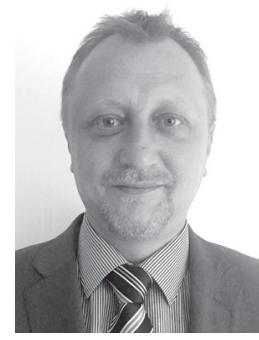

Kardiochirurgia i Torakochirurgia Polska 2016; 13 (3): 195-197

Lung transplantation (LTx) has undergone dramatic development throughout the last years. Today it has become standard treatment of end stage respiratory failure. Based on recent data from the International Society of Heart and Lung Transplantation (ISHLT), almost 4000 procedures are performed worldwide on a yearly basis. However, despite the continuing increase in numbers, there remains a significant gap between the demand and availability of organs. Indeed, based on prognoses of the expected incidence of respiratory diseases, this discrepancy is likely to increase even further. As a result, there is significant mortality on current waiting lists, differing between countries and centers, but up to $16 \%$ and more per year for some diagnoses. One key element affecting this process remains the relatively low utilization of organs, with retrieval rates of a maximum of $28 \%$ for the "Eurotransplant" zone and only $20 \%$ for the USA and UK [1-5].

While the question of safe utilization of the existing donor pool remains open, as does the question of what a transplantable or extended donor organ actually is, we have seen several successful attempts made over the last decade aimed at increasing the organ utilization rate. The first one was the introduction of specialized, adequate donor management during the period between brain stem death (BSD) and organ retrieval. This may have a pivotal role, as it is well known that pathological changes following BSD may have a substantial, detrimental effect on lung function. As a result, numerous guidelines have been published about organ donor management, including the recently introduced UK guidelines: Donor Optimization Extended Care Bundle [6]. This clear and schematic document provides general and organ specific goals of therapy along with advice how to achieve them. It is our experience that the introduction of this protocol resulted in more focused donor management by local intensive care staff. Also, their cooperation with specialist teams dedicated to cardiothoracic donor management provided by transplant centers is, as a result, more open and effective. Proper, pulmonary artery catheter guided therapy is another element. Rational fluid administration, vasopressor and inotropic support to control hemodynamic instability along with steroids to reduce systemic inflammatory responses are important factors in stabilizing and optimizing donors, mitigating the effects of BSD and thus increasing the utilization of organs. Other less difficult maneuvers such as the introduction of lung protective ventilation, patient positioning including upper body elevation and undulating lateral positioning, lung physiotherapy and, when available, bronchoscopy for bronchial toilet are very useful as well. The influence of intensified donor management on the lung retrieval rate has recently been studied in a multicenter analysis by a Spanish group. They demonstrated that the introduction of relatively simple maneuvers, similar to the ones listed above, into donor lung management resulted in more than a doubling $13 \%$ to $28 \%$ - of the retrieval rate within the study group [7]. Even more spectacular results were presented a few years ago by an Australian group. Introduction of a donor management strategy, and close cooperation between donor and recipient centers including involvement of transplant surgeons and physicians, resulted in a higher than $50 \%$ rate of successful lung donation with excellent 1-year results [8].

Another vital question still to be answered conclusively is: which lungs are suitable for transplantation and which are not for an individual recipient? The current so-called standard acceptability criteria, which were somewhat arbitrary and established during earlier developmental phases of clinical LTX, are, in our opinion, very restrictive. Over the years, the vast majority of major centers started to redefine them significantly while simultaneously increasing the number of LTx performed without a substantial detrimental effect on outcomes. Indeed, analysis of United Network for Organ Sharing (UNOS) data published by the Cleveland Clinic group revealed that, based on the original standard criteria, a surprising $56 \%$ of LTX in the US were performed using organs that would be from extended criteria donors (ECD). Currently, only one of the standard criteria has been demonstrated to have an influence on the outcome. And while a history of smoking influences long-term results in the Cleveland study and a recent evaluation in the UK corroborates these data for heavy smokers, mathematical modelling clearly showed that avoiding LTx from smoking donors significantly increases the overall cumulative

Address for correspondence: Bartłomiej Zych, Department of Cardiothoracic Transplantation and Mechanical Circulatory Support, Harefield Hospital, Hill End Road, UB9 6JH Harefield, United Kingdom, e-mail: B.Zych@rbht.nhs.uk

Received: 20.07.2016, accepted: 20.07.2016. 
mortality (including waiting list mortality) $[9,10]$. Our own analysis in a cohort of 237 patients did not show any detrimental effect of a donor smoking history on medium-term survival [11]. In this context, it is important to note that no studies have shown any increase in malignancy after transplantation of lungs procured from smoking donors. This means that lungs from donors even with a history of heavy smoking should not be considered marginal, and stopping performing LTx using these organs would result in a significant increase in mortality on the waiting list and fewer patients benefiting from the procedure.

Our own experience with donors outside standard acceptability criteria confirms that a liberal approach is adequate. A selection of donors based not on these criteria results in a significant increase of the number of LTX with no detrimental effect on outcomes. Data published by our group last year revealed that short- and mid-term outcomes of LTx from donors older than 55 years, with a history of smoking over 20 pack-years and with a $\mathrm{PaO}_{2} / \mathrm{FiO}_{2}$ ratio before retrieval of less than $300 \mathrm{~mm} \mathrm{Hg}$, are comparable to those when standard donor lungs were used. We intentionally defined our extended donor group based on objective, numeric criteria. Donors with abnormal chest X-ray and bronchoscopy only were allocated to the standard donor group to avoid bias caused by a possible different interpretation by the individual operator. Even with this approach, more than $30 \%$ of organs procured and transplanted at our centers came from donors who were ECD by at least one criterion. Hypothetically, if we had not performed LTx using these organs, the number of transplants would have been lower by one third, which would clearly be reflected in waiting list mortality [12]. Thus, based on our and other groups' published results, we strongly advocate a new and more realistic definition of donor selection criteria.

However, while different selection criteria result in increased utilization, it is equally important to adequately match all normal and ECD organs with an appropriate recipient. An important paper published by the Hannover group revealed that ECD lungs allocated to low risk profile recipients via a "rescue allocation scheme" performed very well, thus having an invaluable input in increasing LTX activity. However, there are data and it is our own experience that it is probably unadvisable to match truly marginal donor lungs with high risk recipients [13].

Donation after circulatory death (DCD) has become a vital part of LTx programs in many countries over the last few years. Initial reports, including our own experience, were very promising. But while these initial results were comparable to those after standard after brain-stem death (DBD) donation, further analyses diminished our enthusiasm to a certain degree, as a propensity matched analysis of our long-term results suggested a significant increase in chronic allograft dysfunction (CLAD) in patients receiving lungs from DCD donors. We believe, however, that this is just one cohort observation. It has not been confirmed by others, and data from the Melbourne group (personal communication) suggest a better outcome with less CLAD.
Based on the above, we advocate that more data and longer observation are needed to define the relevance of DCD donation in current clinical practice [14, 15].

The last few years have been a time of dynamic increase in the clinical application of ex-vivo lung perfusion (EVLP). Originally, this technique was introduced into clinical practice by the Lund group when they used it for assessment of lungs from donation after circulatory death (DCD), and then for so-called reconditioning of lungs that had been originally rejected. A modified approach of this technique was introduced by the Toronto group. Today, both protocols are in use: the Swedish one with an open circuit, blood-based perfusate and flow equal to full donor cardiac output; and the Canadian one with a closed circuit, acellular perfusate and flow limited to $40 \%$ of donor cardiac output. Numerous small studies analyzing the effects of EVLP have been published recently, and all of them confirmed the safety and efficacy of this technique, either for assessment or reconditioning of donor lungs considered not acceptable or borderline during the retrieval process [16-18]. Our own experience corroborated these observations. Our small group of 6 patients made an excellent postoperative recovery with 100\% survival until discharge [19]. To date we have performed 31 runs of stationary EVLP and transplanted 11 patients ( $35 \%$ conversion rate). After a median of 3.2 years of observation, survival is $64 \%$, which is lower than average for our center but in line with national UK survival after LTX. In this context, the novel technique of mobile EVLP - using Transmedics' Organ Care System - may become a game changing factor. Initial reports suggested excellent outcomes when the system was used for standard donor lung procurement [20]. Currently we are waiting for the final results of the INSPIRE trial, with an update having been presented at the meeting of the ISHLT in 2016 which seem to demonstrate superiority of mobile EVLP over cold storage in the rate of severe primary graft dysfunction, which is known to be a key risk factor for early death and development of CLAD after LTx. We believe that extracorporeal organ perfusion can be particularly useful for organs obtained from DCD donors. By its nature this kind of donation makes organ assessment and recruitment very limited. The lung function from before withdrawal of treatment (WOT) may differ significantly from function at or after circulatory arrest. This is especially important when long lasting donor hypotension or hypoxia is observed between WOT and death. Doubts about the organ quality in this situation often result in decline of the lung. Thus, the potential for an objective assessment - ex-situ - should dramatically increase the rate of successful retrieval from controlled DCD donors and even pave the way for wider use of uncontrolled DCD donation. However, while it is not a topic of this review, it is worth mentioning that using extracorporeal organ perfusion allowed DCD heart transplantation to be included in the clinical practice. We started to use this approach a year ago, with a good outcome [21]. Based on current and emerging data, we believe that extracorporeal donor organ perfusion, not only of the lungs, may be an important element of the 
future of transplantation. It creates a perspective for better preservation than cold storage, a possibility for much more objective assessment outside the confinements of the organ donor and the donation process, and, last but not least, the potential for therapeutic interventions during the time between retrieval and implantation. An additional effect, albeit not that significant in lung transplantation, is that decreasing the time pressure during the whole transplantation process significantly decreases the logistical complexity and reduces stress on the operating team, both factors well known to influence the outcomes.

In our opinion, the real lung donor pool remains undiscovered. Potentially, many more lungs than currently retrieved remain unused. The ubiquitous introduction of a structured donor management strategy along with new approaches to donation, organ assessment and preservation may pave the way to a further substantial increase in overall lung transplantation activity.

\section{Disclosure}

Authors report no conflict of interest.

\section{References}

1. Yusen RD, Edwards LB, Kucheryavaya AY, Benden C, Dipchand Al, Goldfarb SB, Levvey BJ, Lund LH, Meiser B, Rossano JW, Stehlik J. The Registry of the International Society for Heart and Lung Transplantation: Thirty-second Official Adult Lung and Heart-Lung Transplantation Report - 2015; Focus Theme: Early Graft Failure. J Heart Lung Transplant 2015; 34: 1264-1277.

2. Valapour M, Skeans MA, Heubner BM, Smith JM, Schnitzler MA, Hertz MI, Edwards LB, Snyder JJ, Israni AK, Kasiske BL. OPTN/SRTR 2012 Annual Data Report: lung. Am J Transplant 2014; 14 Suppl 1: 139-165.

3. Organ Donation and Transplantation Activity Report 2014/15. http://nhsbtmediaservices.blob.core.windows.net/organ-donation-assets/pdfs/activity_report_2014_15.pdf

4. Annual Report 2014 Eurotransplant International Foundation. https://www. eurotransplant.org/cms/mediaobject.php?file=ar_2014.pdf

5. Australian Donation And Transplantation Activity Report 2015. http://www. donatelife.gov.au/sites/default/files/Australian\%20Donation\%20and\%20 Transplantation\%20Activity\%20Report\%202015.pdf

6. Donor Optimisation Extended Care Bundle. www.odt.nhs.uk/pdf/dbd_care bundle.pdf

7. Mińambres E, Pérez-Villares JM, Chico-Fernández M, Zabalegui A, DueńasJurado JM, Misis M, Mosteiro F, Rodriguez-Caravaca G, Coll E. Lung donor treatment protocol in brain dead-donors: a multicenter study. J Heart Lung Transplant 2015; 34: 773-780.

8. Snell GI, Griffiths A, Levvey BJ, Oto T. Availability of lungs for transplantation: exploring the real potential of the donor pool. J Heart Lung Transplant 2008; 27: 662-667.
9. Reyes KG, Mason DP, Thuita L, Nowicki ER, Murthy SC, Pettersson GB, Blackstone EH. Guidelines for donor lung selection: time for revision? Ann Thorac Surg 2010; 89: 1756-1764.

10. Bonser RS, Taylor R, Collett D, Thomas HL, Dark JH, Neuberger J; Cardiothoracic Advisory Group to NHS Blood and Transplant and the Association of Lung Transplant Physicians (UK). Effect of donor smoking on survival after lung transplantation: a cohort study of a prospective registry. Lancet 2012; 380: 747-755.

11. Sabashnikov A, Patil NP, Mohite PN, García Saez D, Zych B, Popov AF, Weymann A, Wahlers T, De Robertis F, Bahrami T, Amrani M, Simon AR. Influence of donor smoking on midterm outcomes after lung transplantation. Ann Thorac Surg 2014; 97: 1015-1021.

12. Zych B, García Sáez D, Sabashnikov A, De Robertis F, Amrani M, Bahrami T, Mohite PN, Patil NP, Weymann A, Popov AF, Reed A, Carby M, Simon AR. Lung transplantation from donors outside standard acceptability criteria: are they really marginal? Transpl Int 2014; 27: 1183-1191.

13. Sommer W, Kühn C, Tudorache I, Avsar M, Gottlieb J, Boethig D, Haverich A, Warnecke G. Extended criteria donor lungs and clinical outcome: results of an alternative allocation algorithm. J Heart Lung Transplant 2013; 32: 10651072.

14. Zych B, Popov AF, Amrani M, Bahrami T, Redmond KC, Krueger H, Carby M, Simon AR. Lungs from donation after circulatory death donors: an alternative source to brain-dead donors? Midterm results at a single institution. Eur J Cardiothorac Surg 2012; 42: 542-549.

15. Sabashnikov A, Patil NP, Popov AF, Soresi S, Zych B, Weymann A, Mohite PN, García Sáez D, Zeriouh M, Wahlers T, Choi YH, Wippermann J, Wittwer T, De Robertis F, Bahrami T, Amrani M, Simon AR. Long-term results after lung transplantation using organs from circulatory death donors: a propensity score-matched analysis. Eur J Cardiothorac Surg 2016; 49: 46-53.

16. Steen S, Sjöberg T, Pierre L, Liao Q, Eriksson L, Algotsson L. Transplantation of lungs from a non-heart-beating donor. Lancet 2001; 357: 825-829.

17. Ingemansson R, Eyjolfsson A, Mared L, Pierre L, Algotsson L, Ekmehag B, Gustafsson R, Johnsson P, Koul B, Lindstedt S, Lührs C, Sjöberg T, Steen S. Clinical transplantation of initially rejected donor lungs after reconditioning ex vivo. Ann Thorac Surg 2009; 87: 255-260.

18. Cypel M, Yeung JC, Liu M, Anraku M, Chen F, Karolak W, Sato M, Laratta J, Azad S, Madonik M, Chow CW, Chaparro C, Hutcheon M, Singer LG, Slutsky AS, Yasufuku K, de Perrot M, Pierre AF, Waddell TK, Keshavjee S. Normothermic ex vivo lung perfusion in clinical lung transplantation. N Engl J Med 2011; 364: 1431-1440

19. Zych B, Popov AF, Stavri G, Bashford A, Bahrami T, Amrani M, De Robertis F, Carby M, Marczin N, Simon AR, Redmond KC. Early outcomes of bilateral sequential single lung transplantation after ex-vivo lung evaluation and reconditioning. J Heart Lung Transplant 2012; 31: 274-281.

20. Warnecke G, Moradiellos J, Tudorache I, Kühn C, Avsar M, Wiegmann B, Sommer W, lus F, Kunze C, Gottlieb J, Varela A, Haverich A. Normothermic perfusion of donor lungs for preservation and assessment with the Organ Care System Lung before bilateral transplantation: a pilot study of 12 patients. Lancet 2012; 380: 1851-1858.

21. García-Saez D, Bowles CT, Mohite PN, Zych B, Maunz O, Popov AF, Hurtado A, Raj B, Rahman-Haley SL, Banner NR, Simon AR. Heart transplantation following donor circulatory death in patients bridged to transplant with implantable left ventricular assist devices. J Heart Lung Transplant in press. 